\title{
Nasolaryngoscopy in a Family Medicine Clinic: Indications, Findings, and Economics
}

\author{
Thad Wilkins, MD, Ralph A. Gillies, PhD, April Getz, MD, \\ Dave Zimmerman, $M D, M B A$, and Larry Kang, $M D$
}

Background: Nasopharyngeal complaints are common among patients who present to primary care. Patients with these complaints are often referred for nasolaryngoscopy evaluation to exclude serious conditions such as laryngeal cancer.

Methods: This study is a retrospective case series in which 276 charts of adult outpatients who were referred for nasolaryngoscopy were reviewed. We examined patient demographics, procedure indications and findings, complications, and changes in clinical management.

Results: Nasolaryngoscopy was completed in 273 (98.9\%) patients (mean age, $51.3 \pm 14.6$ years; 71.4\% were women). The most common indications for nasolaryngoscopy were hoarseness $(51.3 \%)$, globus sensation $(32.0 \%)$, and chronic cough (17.1\%); the most common findings included laryngopharyngeal reflux (42.5\%), chronic rhinitis $(32.2 \%)$, and vocal cord lesions $(13.2 \%)$. Three patients (1.1\%) were diagnosed with laryngeal cancer and this diagnosis was significantly associated with a history of smoking $(P=.03)$. No major complications occurred.

Conclusions: We found that nasolaryngoscopy was a safe procedure in the primary care setting, and no major complications occurred in our series. Patients who have ever smoked and complain of hoarseness are at higher risk for laryngeal cancer. An alarming $1 \%$ of patients in our series were diagnosed with laryngeal cancer. This is the first study to define the rates of laryngopharyngeal reflux, vocal cord lesions, and laryngeal cancer among primary care patients. (J Am Board Fam Med 2010;23:591-597.)

Keywords: Laryngoscopy, Laryngeal Neoplasms, Laryngopharyngeal Reflux, Primary Health Care

In the United States in 2008, 12,250 new cases of laryngeal cancer were diagnosed, and an estimated 3,670 deaths occurred from laryngeal cancer. ${ }^{1}$ The death rates from laryngeal cancer did not significantly change from 1990 (2.97 per 100,000) to 2004 (2.24 per 100,000). The 5-year survival rates decreased minimally, from $67 \%$ in 1975 to $64 \%$ in $2003 .{ }^{1}$ Early diagnosis and definitive treatment is the key to in-

This article was externally peer reviewed.

Submitted 29 July 2009; revised 2 March 2010; accepted 8 March 2010.

From the Department of Family Medicine, Medical College of Georgia, Augusta (TW, RAG, AG, DZ); and Eisenhower Army Medical Center, Fort Gordon, GA (LK).

Funding: none.

Conflict of interest: none declared.

Corresponding author: Thad Wilkins, MD, Department of Family Medicine, 1120 15th Street, HB-4032, Medical College of Georgia, Augusta, GA 30912 (E-mail: twilkins@mcg.edu).

\footnotetext{
See Related Commentary on Page 564.
}

creasing survival from laryngeal cancer. Currently, there are no screening guidelines for laryngeal cancer. Although a primary care series of adult smokers aged 40 years and older found a 3\% prevalence of laryngeal cancer, ${ }^{2}$ the prevalence of nasopharyngeal symptoms in primary care is largely unknown.

Family physicians commonly evaluate patients with nasopharyngeal complaints, eg, hoarseness, dysphagia, chronic cough, throat clearing, globus sensation, or chronic sore throat, and some of these patients may have serious conditions such as laryngeal cancer. In a primary care practice research network, the prevalence of dysphagia was $23 \% .^{3}$ In another primary care series, the prevalence of hoarseness was $11 \%$. The association between gastroesophageal reflux disease (GERD) and laryngopharyngeal reflux (LPR) is well established, and extra-esophageal symptoms, eg, hoarseness and globus sensation, improve with acid suppression. ${ }^{4}$ A systematic review found an increased risk of chronic cough in patients with GERD (odds ratio, 1.7; 95\% CI, 1.4-2.1), ${ }^{5}$ and a national cross-sectional postal survey in Scotland found a $31 \%$ prevalence of sore throat. $^{6}$ 
Nasolaryngoscopy is often used to exclude serious medical conditions, eg, laryngeal cancer, in patients with nasopharyngeal complaints, but this procedure is only performed by $6 \%$ of family physicians in the United States. ${ }^{7}$ Nasolaryngoscopy is performed in the office setting with topical anesthesia and nasal decongestants. In 1998 an initial study of nasolaryngoscopies performed by family physicians $(n=66)$ reported a mean examination time of 4.6 minutes, and the procedure was well tolerated by patients. ${ }^{8}$ In a subsequent study, 210 patients were evaluated by nasolaryngoscopies performed by family physicians; $90 \%$ of these cases resulted in changes in diagnosis or management after the procedure; however, both of these case series were published 20 years ago. In this article and in our study we define "nasolaryngoscopy" to mean a nasopharyngoscopy (endoscopy of the nose and nasopharynx) or a nasolaryngoscopy (endoscopy of the nose, nasopharynx, and larynx). The purpose of this study was to determine the common indications, findings, the rate of laryngeal cancer, and quantify the economics of nasolaryngoscopy in an urban, university-based family medicine clinic in the Southeastern United States.

\section{Methods}

This retrospective chart review examined the medical records of 276 adult, English-speaking, nonemergent, consecutive outpatients older than 18 years of age who had a nasolaryngoscopy performed in a university-based family medicine clinic. Our family medicine clinic has approximately 30,000 patient visits per year, $47 \%$ of whom are African American and 66\% are women. Nasolaryngoscopy was deemed necessary by the patient's primary care physician based on nasopharyngeal complaints and was performed as part of a medical evaluation of these complaints. All patients referred for this procedure were scheduled without exclusion criteria. We did not track data about the percent of patients who missed or canceled their appointment for this procedure. After they provided informed consent, patients were anesthetized using a method we have described previously. ${ }^{9}$ The procedures in this study were performed by or supervised by the lead author (TW) between February 2002 and April 2008 using either XEF-140Y1 gastrointestinal videoscope or ENF-P3 laryngoscope (Olympus America, Inc., Melville, NY). The XEF-
$140 \mathrm{Y} 1$ has an outer diameter of $4.0 \mathrm{~mm}$ at the insertion tube, a working length of $600 \mathrm{~mm}$, a bending section that can be maneuvered upward 180 degrees and downward 90 degrees (with no right or left deflection), and a field of view of 120 degrees. The ENF-P3 has an outer diameter of 3.7 $\mathrm{mm}$ at the insertion tube, a working length of 300 $\mathrm{mm}$, a bending section that can be maneuvered upward 130 degrees and downward 130 degrees (with no right or left deflection), and a field of view of 85 degrees. The study was approved by the Medical College of Georgia's institutional review board.

A list of patients was obtained by a clinical query of our electronic medical record using "nasolaryngoscopy" as a search term and by reviewing an established list of procedures that is maintained by the procedure nurse in the family medicine clinic. We reviewed the chart records for demographic data, indications, findings, and need for further consultation or evaluation. Complications were also noted.

\section{Data Analysis}

The primary analyses for the study included descriptive statistics of the indications and findings for nasolaryngoscopy. Subsequent $\chi^{2}$ analyses examined the influence of the following independent factors on indications and findings: age, sex, ethnicity, and alcohol and tobacco use. SPSS software version 11 (SPSS, Inc., Chicago, IL) was used for all analyses.

\section{Results}

Two hundred seventy-six patients were included in this case series. The mean age of the patients was 51.3 years $( \pm 14.6) ; 71.4 \%$ were women, $48.1 \%$ were white, and $46.9 \%$ were black. Table 1 presents the demographics of patients in this series. The most common indications for the nasolaryngoscopy were hoarseness (51.3\%), globus sensation (32.0\%), chronic cough $(17.1 \%)$, chronic rhinitis $(13.5 \%)$, and sore throat (10.9\%). Table 2 lists the common indications for nasolaryngoscopy as well as how each indication varied across demographic groups. Chronic rhinitis and chronic sinusitis were more common in patients $<50$ years of age compared with patients $>50$ years old $(P=.007$ and $P=.02$, respectively). Dysphagia was more common among patients who drank alcohol (12.8\%) 
Table 1. Demographics of Patients Referred for Nasolaryngoscopy $(\mathrm{n}=276)$

\begin{tabular}{lc}
\hline Factor & \\
\hline Age (mean years \pm SD) & $51.3 \pm 14.6$ \\
Female sex & $197(71.4)$ \\
Race & \\
Black & $121(46.9)$ \\
White & $124(48.1)$ \\
Other & $13(4.7)$ \\
Alcohol use & \\
Current (yes) & $66(24.7)$ \\
Past (yes) & $83(31.1)$ \\
Tobacco use & \\
Current & $70(25.8)$ \\
Ever & $104(38.4)$ \\
\hline
\end{tabular}

Values provided as $\mathrm{n}(\%)$ unless otherwise indicated.

compared with those that did not drink $(3.3 \% ; P=$ $.003)$. Vocal cord lesions were more common among smokers $(6.7 \%)$ versus nonsmokers $(0.6 \%$; $P=.004)$.

Nasolaryngoscopy was completed in 273 (98.9\%) of the patients. Three patients could not tolerate the procedure because of pain. Minor complications occurred in 3 procedures (1.1\%): 2 patients experienced pain but the procedure was completed and 1 patient experienced mild epistaxis that was controlled with pressure. Most of the procedures were performed nasally $(98.6 \%)$, with 3 procedures performed orally $(1.1 \%)$ and one procedure completed via both routes $(0.3 \%)$. Although we did not record procedure times, in our experience procedure times are generally $\leq 5$ minutes. A resident assisted with 154 procedures $(55.8 \%)$. In our clinic, $80 \%$ of procedures were nasolaryngoscopy and $20 \%$ of procedures were nasopharyngoscopy.

Nasolaryngoscopy was normal in 35 patients $(12.8 \%)$. The most common findings from the nasolaryngoscopy were LPR (42.5\%); chronic rhinitis (32.2\%); and vocal cord lesions, eg, granuloma or polyp (13.2\%). Table 3 lists the common findings. A new medication, eg, proton pump inhibitor, antihistamine, or intranasal steroid, was added for 140 patients $(50.7 \%)$ and in 9 patients $(3.3 \%)$ a medication was discontinued (eg, $\mathrm{H}_{2}$-blocker). Seventytwo patients received a referral to otolaryngology (26.1\%). Table 4 shows management changes after the nasolaryngoscopy.

Of the 72 patients who were referred to otolaryngology, 3 patients $(1.1 \%)$ were diagnosed with

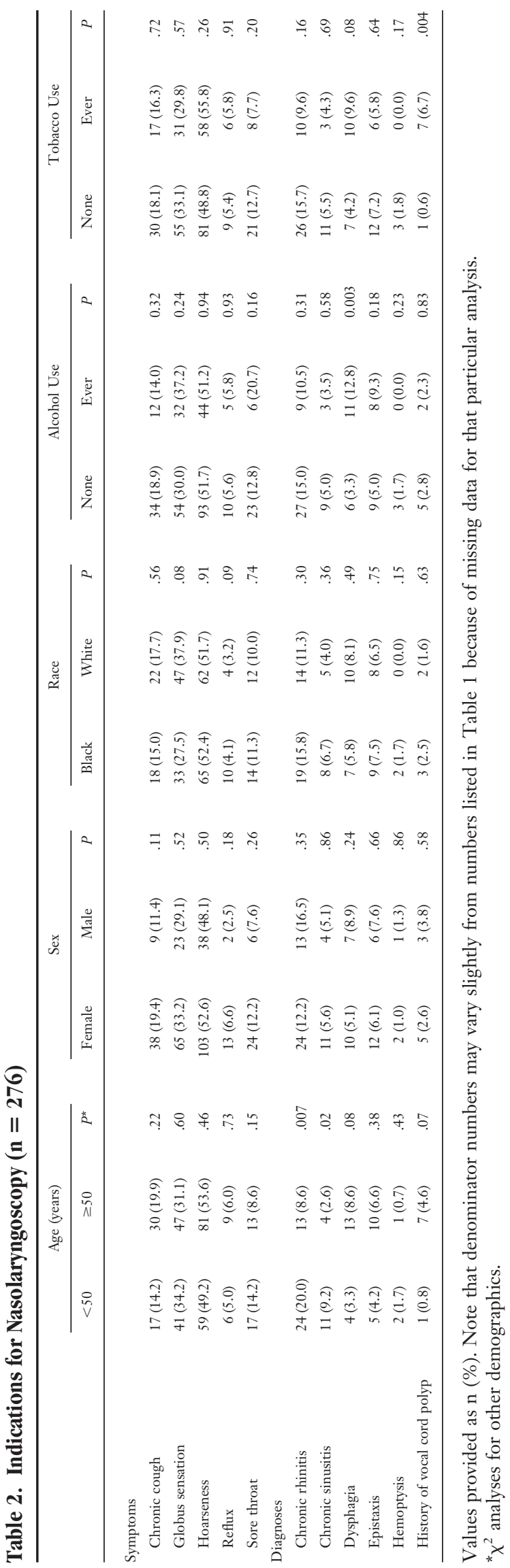

doi: 10.3122/jabfm.2010.05.090186

Nasolaryngoscopy in a Family Medicine Clinic 593 
Table 3. Findings from Completed Nasolaryngoscopy

$(\mathrm{n}=273)^{*}$

\begin{tabular}{lr}
\hline Findings & $\mathrm{n}(\%)^{\dagger}$ \\
\hline Laryngopharyngeal reflux & $116(42.5)$ \\
Chronic rhinitis & $88(32.2)$ \\
Other $^{\ddagger}$ & $84(30.8)$ \\
Vocal cord lesion $_{\text {Nasal polyps }}$ & $36(13.2)$ \\
Other pharyngeal lesion & $10(3.7)$ \\
Precancerous lesion & $8(2.9)$ \\
Laryngeal mass & $5(1.8)$ \\
Normal findings & $2(0.7)$ \\
\hline
\end{tabular}

*Three of the 276 patients did not complete the entire nasolaryngoscopy procedure.

${ }^{\dagger}$ Percentages do not sum to $100 \%$ because patients may have had more than one finding.

‡"Other” includes tissue hypertrophy, acute inflammation, aspiration, eustachian tube dysfunction, candidal infection, septal perforation, bony spur, aphthous ulcer, vocal cord dysfunction, prominent vessel, and hemangioma.

laryngeal cancer and $9(3.3 \%)$ were diagnosed with vocal cord lesions. One hundred sixteen patients were diagnosed with LPR using nasolaryngoscopy, and an additional 18 patients were classified with LPR during subsequent testing, for a total of 134 $(48.5 \%)$. Table 5 provides referral outcomes.

$\chi^{2}$ analysis was used to determine which factors were significantly associated with referral out-

Table 4. Management Changes After Nasolaryngoscopy $(\mathrm{n}=\mathbf{2 7 6})$

\begin{tabular}{lc}
\hline Management & $\mathrm{n}(\%)^{*}$ \\
\hline Medication changes & \\
Added & $140(50.7)$ \\
Deleted & $9(3.3)$ \\
Referrals & \\
Otolaryngology & $72(26.1)$ \\
Gastroenterology & $8(2.9)$ \\
Other diagnostic tests & \\
CAT scan & $18(6.5)$ \\
Barium swallow & $14(5.1)$ \\
Esophagogastroduodenoscopy & $12(4.3)$ \\
Repeat nasolaryngoscopy & $5(1.8)$ \\
Other & \\
No changes to therapeutic plan & $16(5.8)$ \\
\hline
\end{tabular}

*Percentages do not sum to $100 \%$ because patients may have had more than one change.

t"Other" includes sleep study, allergy/immunology consult, manometry, pillcam, and transnasoesophagoscopy.

CAT, computed axial tomography.
Table 5. Referral Outcomes*

\begin{tabular}{lcc}
\hline & $\begin{array}{c}\text { Referred patients } \\
(\mathrm{n}=127) \\
(\mathrm{n}[\%])\end{array}$ & $\begin{array}{c}\text { Overall } \\
(\mathrm{n}=276)(\%)\end{array}$ \\
\hline $\begin{array}{l}\text { Referral Outcome } \\
\begin{array}{l}\text { Laryngeal cancer } \\
\text { (confirmed by biopsy) }\end{array}\end{array}$ & $3(2.4)$ & $(1.1)$ \\
$\begin{array}{l}\text { Vocal cord lesion } \\
\text { Laryngopharyngeal reflux } \\
\text { Other }\end{array}$ & $9(7.1)$ & $(3.3)$ \\
\hline
\end{tabular}

*Patient may have more than one outcome.

${ }^{\dagger}$ One hundred twenty-seven patients were referred for follow-up assessment by otolaryngology or gastroenterology or for other diagnostic tests.

‡" Other outcome" includes vocal cord granuloma, sinonasal disease, nodules, vocal cord polyp, esophageal cancer with metastasis, parotid adenoma, small cell lung cancer, enlarged thyroid, esophagitis, and gastritis.

comes. Laryngeal cancer was associated with previous or current tobacco use $(P=.03)$. Vocal cord lesions were associated with hoarseness $(P=.003)$ and previous or current tobacco use $(P<.0001)$. LPR was associated with hoarseness $(P=.009)$ and sore throat $(P=.03)$.

\section{Discussion}

In our case series, 276 patients underwent nasolaryngoscopy for various nasopharyngeal symptoms, including hoarseness, globus sensation, chronic cough, chronic rhinitis, sore throat, epistaxis, dysphagia, and reflux. Laryngeal cancer should be excluded in patients with persistent or chronic nasopharyngeal symptoms, especially in those patients who have risk factors for developing cancer, which include tobacco and alcohol use, GERD, and occupational exposure. The prevalence of laryngeal cancer in our series was an alarming $1 \%$. This finding is of particular importance for patients who present with nasopharyngeal complaints like hoarseness, but this finding may not be generalizable. We were interested in determining the rate of tobacco use among the patients in our series. Nationally, $24 \%$ of individuals self-report tobacco use ${ }^{10}$ compared with $26 \%$ in our study; however, self-report of tobacco use has been shown to be an underestimation of the true smoking prevalence. ${ }^{11}$

The overall prevalence of LPR was $48.5 \%$. LPR is a condition in which gastric contents flow in a retrograde fashion and contact the tissues of the upper aerodigestive tract. LPR is associated with 
hoarseness, cough, globus sensation, refractory asthma, laryngeal ulcers and granulomas, subglottic stenosis, and laryngeal cancer. Physiologic barriers protect the oropharyngeal tract from reflux injury; however, the epithelium of the respiratory tract is sensitive to damage when these mechanisms fail. A recent Cochrane review found insufficient evidence to support acid suppression for the treatment of hoarseness ${ }^{12}$; however, acid suppression with proton pump inhibitors is effective for decreasing the symptoms of LPR and possibly decreasing the risk of complications from LPR.

Common Current Procedural Technology (CPT) billing codes for nasolaryngoscopy include 31575 (laryngoscopy, flexible fiber optic) and 92511 (nasopharyngoscopy with endoscope). Typical Medicare and private insurance reimbursement rates are $\$ 67.75$ and $\$ 230.00$, respectively, for CPT code 31575 and $\$ 53.28$ and $\$ 65.37$, respectively, for CPT code 92511. The fixed equipment necessary to perform nasolaryngoscopy in primary care include a nasolaryngoscope, light source, guide cable, and rigid eyepiece for viewing, and startup costs are estimated at $\$ 8,675$ to $\$ 10,850$. A service contract can be added for $\$ 2,000$ per year, which yields a total fixed startup cost for the first year of $\$ 10,675$ to $\$ 12,850$, assuming a cash purchase. In our clinic, $80 \%$ of procedures billed are laryngoscopy flexible fiber optic (31575), and with our payor distribution mix (80\% private insurance and $20 \%$ Medicare), it would take approximately 76 procedures to break even during the first year. Although we did not calculate the break-even point for subsequent years, it would be substantially less (including maintenance and cleaning costs of equipment). Table 6 outlines an estimation of breakeven points, assuming various mixtures of payor sources and ratios of CPT billing codes. This rudimentary cost model assumes labor to be a sunk cost and no variable costs (such as cleaning costs and supplies such as lidocaine and afrin). Further, opportunity costs are considered to be zero.

Nasolaryngoscopy is often done with local anesthesia and nasal decongestants, without the need for sedation. It is a brief procedure with short recovery time, and patients are able to return to their usual activity, including working and driving, immediately after the procedure. Another inherent advantage of nasolaryngoscopy is that results are immediately available, and the endoscopist can review results with the patient after the procedure. Another potential advantage for patients and physicians is that this is a brief in-office procedure ( $<5$ minutes).

Table 6. Estimated Breakeven Points Assuming Various Ratios of Payor Sources and Current Procedural Technology (CPT) Billing Codes

\begin{tabular}{|c|c|c|c|c|c|c|}
\hline \multirow[b]{2}{*}{ Relative CPT Billing Code Ratio* } & \multicolumn{6}{|c|}{ Relative Payor Ratio (Medicare/Private Insurance) } \\
\hline & $0 \% / 100 \%$ & $20 \% / 80 \%$ & $40 \% / 60 \%$ & $60 \% / 40 \%$ & $80 \% / 20 \%$ & $100 \% / 0 \%$ \\
\hline $\begin{array}{l}0 \% 92511 \\
100 \% 31575\end{array}$ & 56 & 66 & 78 & 97 & 129 & 190 \\
\hline $\begin{array}{l}20 \% 92511 \\
80 \% 31575\end{array}$ & 66 & 76 & 90 & 110 & 141 & 199 \\
\hline $\begin{array}{l}40 \% 92511 \\
60 \% 31575\end{array}$ & 79 & 90 & 105 & 125 & 156 & 208 \\
\hline $\begin{array}{l}60 \% 92511 \\
40 \% \\
31575\end{array}$ & 98 & 111 & 126 & 147 & 175 & 218 \\
\hline 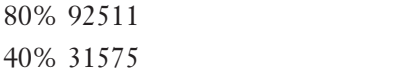 & 131 & 143 & 158 & 176 & 199 & 229 \\
\hline $\begin{array}{l}100 \% 92511 \\
0 \% 31575\end{array}$ & 197 & 205 & 213 & 222 & 231 & 242 \\
\hline
\end{tabular}

Data are presented as Q values at different payor mix and CPT code ratios. Q equals the no. of procedures required to breakeven at the end of the first year, calculated as:

$\mathrm{Q}=\mathrm{T} /\left(\mathrm{N}\left[\left(\mathrm{M}^{*} 53.28\right)+\left(\mathrm{P}^{*} 65.37\right)\right]+\mathrm{L}\left[\left(\mathrm{M}^{*} 67.75\right)+\left(\mathrm{P}^{*} 230\right)\right]\right)$

where $\mathrm{N}$ equals the percentage of procedures performed coded as nasopharyngoscopy; $\mathrm{L}$ equals the percentage of procedures performed coded as laryngoscopy; $\mathrm{M}$ equals the percentage of procedures covered by Medicare insurance; $\mathrm{P}$ equals the percentage of procedures covered by private insurance; and $\mathrm{T}$ equals the total estimated startup costs for the first year $(\$ 12,850)$.

${ }^{*}$ CPT code 92511: Nasopharyngoscopy with endoscope; CPT code 31575: Laryngoscopy, flexible fiber optic. 
Nearly $75 \%$ of patients in this study had rhinitis or LPR, which can be managed by family physicians without the need for specialty referral. Undergoing the procedure in the primary physician's office has a number of other potential advantages. It allows the primary care physician to be directly involved with patient care, eliminates the wait time for the patient to be evaluated by another physician, may decrease cost by decreasing evaluation by specialists, improves convenience for the patient with fewer office visits, decreases anxiety awaiting procedure and results, and improves compliance with completing the procedure. The apparent disadvantages of nasolaryngoscopy include the somewhat narrow field of view, less maneuverability than other endoscopes with up/down and left/right controls, lack of biopsy or suction capability, and missed diagnosis (eg, missed laryngeal cancer).

Our study was done at an academic universitybased medical center, which may not represent the typical primary care practices from a racial (primarily white and black patients), social (alcohol and tobacco use), or economic (insurance and payer mix) point of view. There was an over representation of women in our case series (71.4\%); the most likely explanation is that it reflects the higher percentage of women $(66 \%)$ seen in our clinic. Other possible reasons for more women in our series are that more women discuss nasopharyngeal complaints with their physicians or more women agree to nasolaryngoscopy compared with men. We did not record the time of the procedure. No information was reported about the total patient population that was initially referred for nasolaryngoscopy versus those who were seen and had a procedure. Patient tolerance of the procedure, pain, and level of anxiety and the patient's willingness to undergo a repeat nasolaryngoscopy were not evaluated in our study. In addition, the effectiveness and accuracy of nasolaryngoscopy performed by primary care physicians versus specialists has not been evaluated. Training of family medicine residents in nasolaryngoscopy is not standardized and varies by residency program. Our residency training program requires a minimum of 10 procedures, evaluation of technical skills by an attending physician, and a written examination.

Future studies should assess training variation among family medicine residency programs and competency of graduates to perform nasolaryngoscopy. Additional studies might address the diag- nostic accuracy of nasolarynoscopy performed by family physicians compared with a gold standard. Another interesting study might examine the cost effectiveness of managing patients with nasopharyngeal complaints in primary care versus specialty care. Lastly, a future study should address the natural history of patients with nasopharyngeal complaints in primary care and those that progress to laryngeal cancer.

\section{Conclusion}

In our series there was a $1 \%$ prevalence of laryngeal cancer among patients who were referred for nasolaryngoscopy. Although there is no screening recommendation for laryngeal cancer, those patients at high risk, eg, smokers with chronic hoarseness or throat pain, should be considered for nasolaryngoscopy. LPR was a common diagnosis among patients in our case series. This is an important diagnosis because of the risk of complications (vocal cord granuloma, laryngeal ulcer, etc), and these patients see improvement of their symptoms with aggressive acid suppression. We also found that nasolaryngoscopy is a safe procedure when performed in the primary care setting. In our experience with training family medicine residents in nasolaryngoscopy, technical expertise is rapidly acquired in as few as 10 supervised procedures. Although the procedure is technically easy to learn, confidence in accurately making a diagnosis and ruling out cancer can be more challenging. With only $6 \%$ of family physicians performing nasolaryngoscopy, this procedure is an important but underutilized procedure in family medicine. Given our findings and potential revenue, can family physicians afford not to offer nasolaryngoscopy?

\section{References}

1. Jemal A, Siegel R, Ward E, et al. Cancer statistics, 2008. CA Cancer J Clin 2008;58:71-96.

2. Prout MN, Sidari JN, Witzburg RA, Grillone GA, Vaughan CW. Head and neck cancer screening among 4611 tobacco users older than forty years. Otolaryngol Head Neck Surg 1997;116:201-8.

3. Wilkins T, Gillies RA, Thomas AM, Wagner PJ. The prevalence of dysphagia in primary care patients: a HamesNet Research Network study. J Am Board Fam Med 2007;20:144-50.

4. Qua CS, Wong CH, Gopala K, Goh KL. Gastrooesophageal reflux disease in chronic laryngitis: prevalence and response to acid-suppressive therapy. Aliment Pharmacol Ther 2007;25:287-95. 
5. Hungin AP, Raghunath AS, Wiklund I. Beyond heartburn: a systematic review of the extra-oesophageal spectrum of reflux-induced disease. Fam Pract 2005;22:591-603.

6. Hannaford PC, Simpson JA, Bisset AF, Davis A, McKerrow W, Mills R. The prevalence of ear, nose, and throat problems in the community: results from a national cross-sectional postal survey in Scotland. Fam Pract 2005;22:227-33.

7. American Academy of Family Physicians. Table 64. Performance of diagnostic procedures in family physicians practices, July 2008. Available at http://www. aafp.org/online/en/home/aboutus/specialty/facts/64. html. Accessed 12 May 2009.

8. Corey GA, Hocutt JE Jr, Rodney WM. Preliminary study of rhinolaryngoscopy by family physicians. Fam Med 1988;20:262-5.
9. Wilkins T, Gillies RA. Office-based unsedated ultrathin esophagoscopy in a primary care setting. Ann Fam Med 2005;3:126-30.

10. Klein JD, Thomas RK, Sutter EJ. Self-reported smoking in online surveys: prevalence estimate validity and item format effects. Med Care 2007;45: 691-5.

11. Gorber SC, Schofield-Hurwitz S, Hardt J, Levasseur G, Tremblay M. The accuracy of self-reported smoking: a systematic review of the relationship between self-reported and cotinineassessed smoking status. Nicotine Tob Res 2009; 11:12-24.

12. Hopkins C, Yousaf U, Pedersen M. Acid reflux treatment for hoarseness. Cochrane Database Syst Rev 2006;(1):CD005054. 